\title{
ELF F
}

\section{Rare lung cancers}

There are several different kinds of lung cancer, often referred to as lung cancer subtypes. Some of these occur more often than others. In this factsheet we will specifically look at the subtypes of cancers that do not happen very often and are considered 'rare'.

People's experiences of these rarer types of lung cancer will generally be similar to those of people with more common forms of the condition. However, there are some differences in treatment and outlook. Certain types of lung cancer also need different tests before they can be diagnosed.

This factsheet aims to outline the characteristics of some rare lung cancers, and highlight where each type of lung cancer may be different.

\section{General points}

\section{Diagnosis}

Generally the process of being diagnosed with lung cancer is as follows:

An X-ray and a computerised tomography (CT) scan (where your body is X-rayed at a number of angles before a computer puts together a detailed image) of your chest will first be done to show if there is a lung tumour.

You will then need to have some tests that can show how far the cancer has spread. This process is called staging and could involve further CT scans of the abdomen (stomach area) and brain, or a positron emission tomography CT (PET CT) scan (where a CT scan is combined with a PET scan, which involves a small amount of radioactive dye being injected into your veins to show up anything abnormal in your tissues).

Finally, your doctor will confirm the diagnosis of cancer by taking some samples of the cells from your tumour and testing them (this is called a biopsy).

\section{Treatment}

The lung cancers covered in this factsheet are rare, so few clinical trials to test and establish specific treatments for them have taken place. Therefore, the type 
of treatment you might be offered will be based on your particular situation, expert opinion and the particular treatments available in your country.

\section{Outlook}

Lung cancer is a serious illness and, unfortunately, the prognosis for rare lung cancers is not very good overall. However, lots of work is being done to develop new treatments.

This factsheet will give information on the outlook for each of these rare lung cancers based on the data currently available. However, it is important to remember that everyone is different, and that you may not have the same reaction to a treatment as another person. You should see your prognosis as a guide.

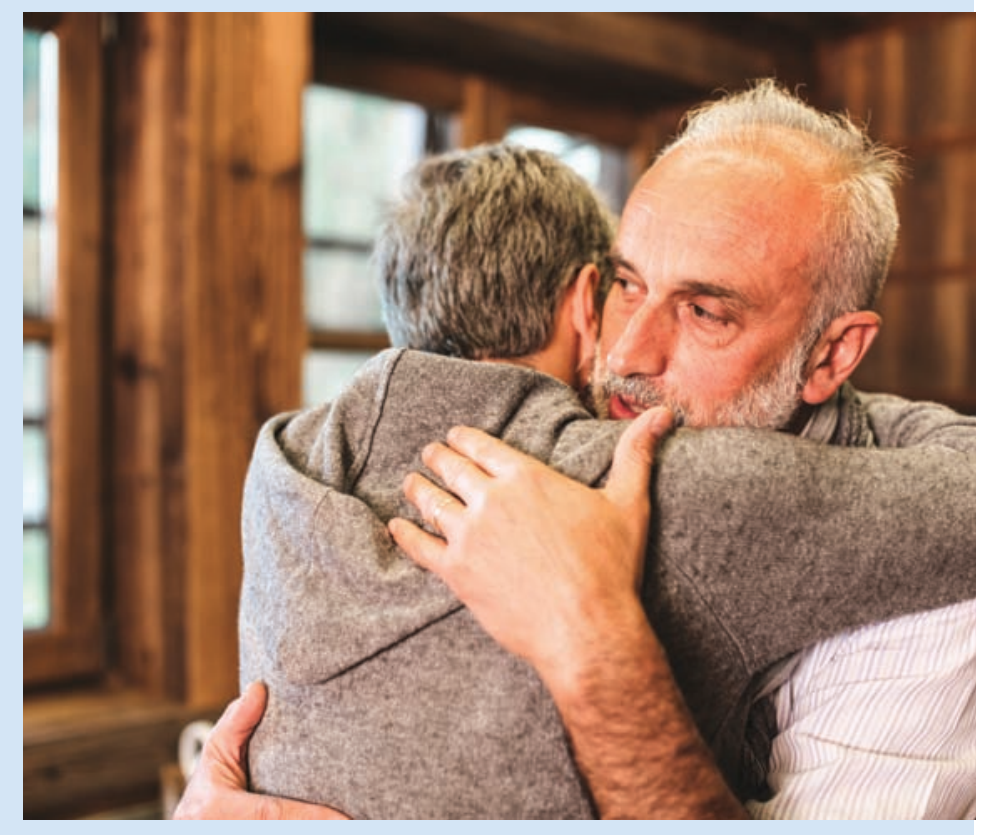

Most of the outlook information in this factsheet is given in terms of a '5-year survival rate'. This term is often used by healthcare professionals, and refers to the number of people studied in research who lived for 5 years or more after being diagnosed with this type of lung cancer.

\section{Adenosquamous carcinoma of the lung}

Adenosquamous carcinoma is a type of tumour that is made up of two of the main tumour types (adenocarcinoma and squamous). It is a rare subtype of non-small cell lung cancer, making up $0.4-4 \%$ of all lung cancer cases.

\section{Diagnosis}

The tests used to diagnose adenosquamous carcinoma are no different from the ones used in other types of lung cancer.

\section{Treatment}

Treatment of adenosquamous carcinoma can differ from more common types of lung cancer as the mix of the two different types of tumour means that a certain chemotherapy drug, pemetrexed (commonly used in adenocarcinomas), will not be effective. Treatment will normally involve chemotherapy drugs that specifically target the squamous component of the tumour, such as cisplatin.

\section{Outlook}

People with adenosquamous carcinomas tend to survive for shorter lengths of time 
than those with pure adenocarcinomas or squamous cell carcinomas of the lung, regardless of how advanced the cancer is at the time of diagnosis or surgical removal of the tumour. The proportion of the adenocarcinoma/squamous cell component (i.e. how much of the tumour is adenocarcinoma and how much is squamous cell carcinoma) does not seem to affect the outcome. For a significant number of people with adenosquamous carcinomas, the cancer spreads (metastasises) to the brain.

\section{Large cell neuroendocrine carcinoma}

Large cell neuroendocrine carcinoma is a subtype of non-small cell lung cancer, representing about $3 \%$ of all lung cancers. It is a neuroendocrine tumour, meaning it develops from cells of the nervous and endocrine (hormonal) systems.

\section{Diagnosis}

Large cell neuroendocrine carcinoma is diagnosed in the same way as other types of lung cancer. However, since neuroendocrine tumours are sometimes harder to pick up on PET CT scan, it is likely that CT scans will be used to assess the extent of the cancer and in the follow-up process.

\section{Treatment}

Most aspects of treatment of this form of cancer are the same as more common forms of lung cancer.

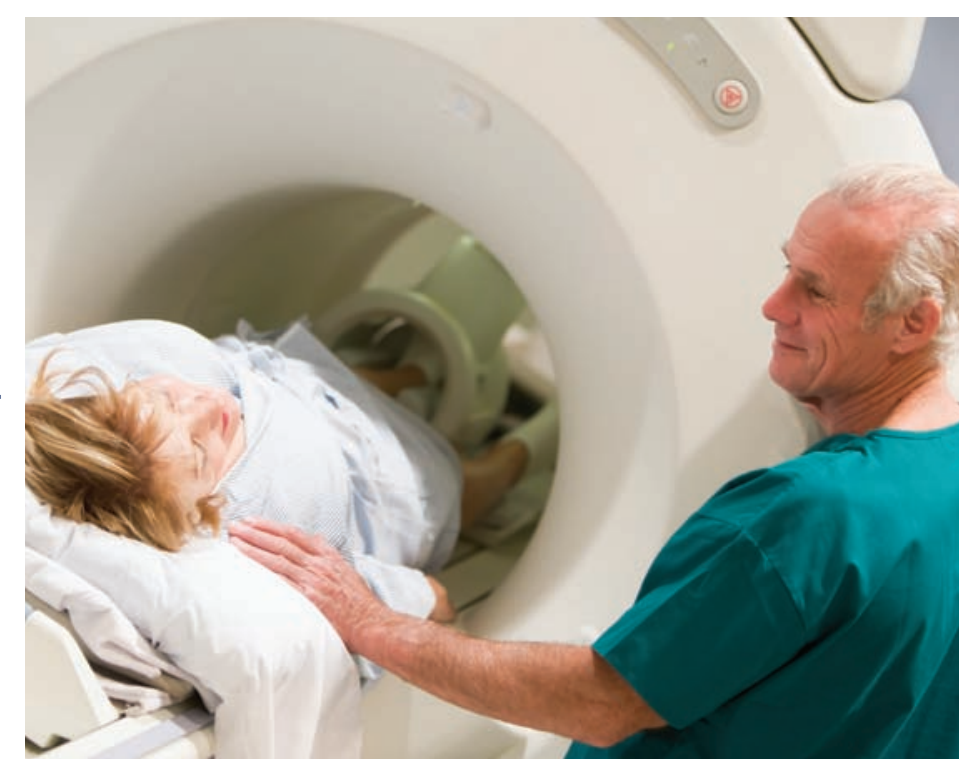

If you are fit enough for surgery, it is likely that you will have an operation to remove the tumour. You may receive a form of chemotherapy containing platinum-based drugs in order to shrink the tumour as much as possible before the operation in order to make it easier to remove.

If your tumour is more advanced, you will be given chemotherapy similar to the one used to treat small cell lung cancers. While good response rates have been shown for this treatment, overall survival rates range from 8 to 16 months.

\section{Outlook}

Recent data showed the following:

- $27.4 \%$ of people live for another 5 or more years after their diagnosis without the cancer returning

- $35.3 \%$ of people live for another 5 or more years after their diagnosis 


\section{Salivary gland-type lung carcinoma}

Salivary gland-type lung carcinomas account for $0.1-0.2 \%$ of all lung cancers. They include adenoid cystic carcinomas and mucoepidermoid carcinomas of the lung. Salivary gland-type carcinomas were previously known as bronchial adenomas. They are thought to come from the glands of the airways, and the tumours are usually in the airways.

\section{Diagnosis}

The symptoms and process of diagnosis for salivary gland-type lung carcinoma are similar to those for common types of lung cancer.

\section{Treatment}

Salivary gland-type lung carcinomas are usually surgically removed. On some occasions, this operation may be accompanied by chemotherapy.

\section{Outlook}

Studies report that survival rates for people with salivary gland-type lung carcinomas that had their tumour surgically removed are as follows:

- 3-year survival rate: $82 \%$

- 5-year survival rate: $70 \%$

- 10-year survival rate: $63 \%$

\section{Sarcomatoid carcinoma of the lung}

Between 0.3 and $3 \%$ of all non-small lung carcinomas are sarcomatoid carcinomas.

Most people diagnosed with this type of cancer are ex- or current heavy smokers. However it is worth noting that a group of these people report that they have also been exposed to asbestos, so it is believed that there could be a link between this type of cancer and asbestos.

It is thought that there could be an overlap between sarcomatoid carcinoma of the lung and another type of lung cancer called mesothelioma that starts in the covering of the lungs (pleura) and is caused by exposure to asbestos.

Like mesothelioma, sarcomatoid carcinoma usually develops in the pleura and has a very slow onset and progression.

\section{Diagnosis}

Diagnosis of sarcomatoid carcinoma usually follows the same course as that for other non-small lung cancers and mesothelioma. 


\section{Treatment}

If your tumour is just in one place and has not spread, it is most likely that you will be treated by having it removed surgically.

If your cancer has spread, you will be treated with standard, mostly platinum-based, chemotherapy drugs.

Recent preliminary research findings show that a specific protein called c-Met drives the development of sarcomatoid carcinoma.

Clinical drug trials related to this finding are currently taking place. This could eventually result in new treatments for sarcomatoid carcinoma that target c-Met.

\section{Outlook}

Sarcomatoid carcinoma is very aggressive. Overall, $20 \%$ of people with sarcomatoid carcinoma lived for 5 years or more after their diagnosis.

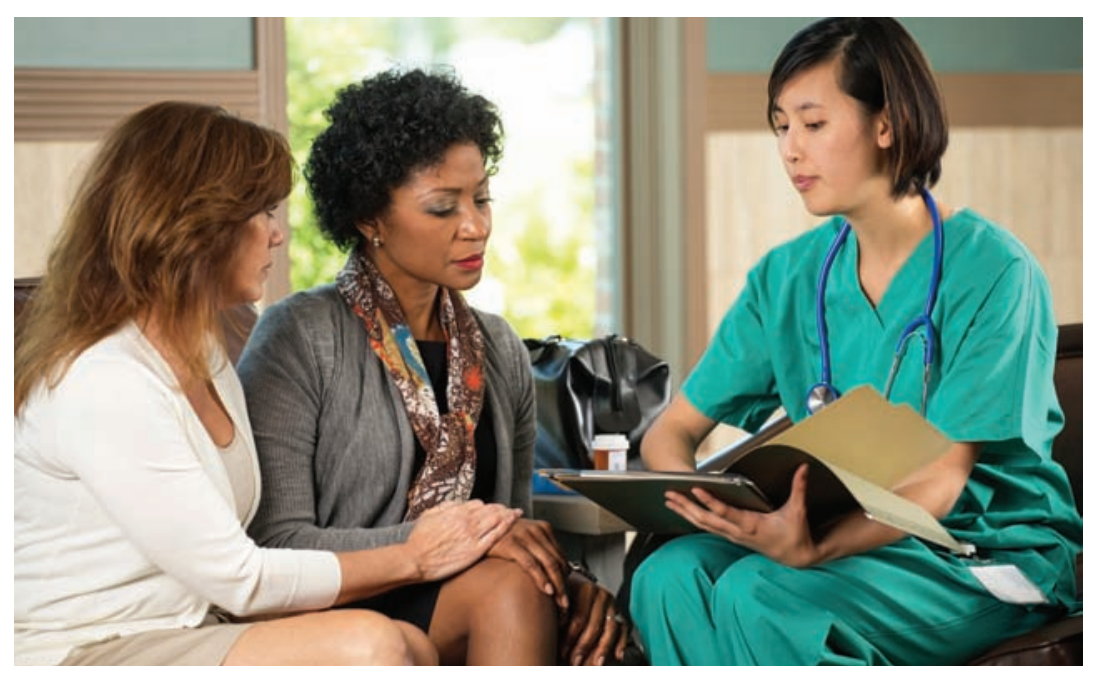

\section{Granular cell lung tumours}

Granular cell lung tumours are extremely rare, making up $0.2 \%$ of all lung tumours. It is even rarer they have malignant (cancerous) cells.

These are usually small, firm, solitary nodules (lumps) found either blocking or surrounding the airways and causing them to narrow.

\section{Diagnosis}

Granular cell lung tumours are diagnosed in the same way as other lung cancers.

\section{Treatment}

Experts have been unable to establish the best treatment for endobronchial granular cell tumours due to a lack of studies. Current treatment options include removal of the tumour via surgery, endoscopy (for which a long instrument called a bronchoscope which has a camera on the end is put down your throat to where the tumour is), fulguration (in which an electric current is used to destroy the tumour), or laser treatment. Surgical removal has the highest cure rates. 


\section{Carcinoids}

Lung carcinoids are a type of tumour that relate to the neuroendocrine system. They are very different to the other types of tumour listed in this factsheet, and account for approximately $1-6 \%$ of all lung tumours. About $25 \%$ of people with carcinoid tumours of the lung do not have symptoms at the time of discovery.

Carcinoid tumours can be detected through a simple chest X-ray or chest $\mathrm{CT}$ scan like all lung cancers. Unlike other types of lung cancer, PET CT scans are not sensitive enough to pick up on them or see whether they have spread. Therefore, if you are suspected of having a carcinoid tumour, you will be given a test called an octreotide scan which uses a different radioactive dye.

\section{Typical carcinoids}

The average age of people who have typical carcinoid tumours is 40-50 years, but they have been reported in virtually every age group, even in teenagers.

Typical carcinoids usually grow quite slowly and rarely spread (metastasise) beyond the lungs. They are usually treated by being removed surgically. This could involve the following procedures:

- Segmentectomy: where either just the tumour and a bit of the surrounding healthy tissue is removed

- Lobectomy: where the whole segment of the lung where the tumour is located is removed

On the rare occasions when a tumour is larger, the following surgical procedures are used:

- Bilobectomy: a part of the lung bigger than the segment where the tumour is located is removed

- Pneumonectomy: the entire lung is removed

$80-90 \%$ of typical carcinoids develop in the small airway, while about $10 \%$ arise in the big airway, and around 10\% appear around the edges of the lung.

As these tumours are usually located in the airways, bronchoscopy (for which an instrument called a bronchoscope which has a camera on the end is put down your throat to where the tumour is) is the most common way to get a sample (biopsy) and confirm the nature of these tumours.

If you are unable to have the tumour surgically removed (due to its size, problems with your lung function or having other significant health issues), your tumour may 
be taken out via an interventional bronchoscopy, which can be done in a specialist centre. The procedure involves a camera being placed down your lungs while the tumour is cut into little pieces and removed, either completely or a large part. This is usually done with laser/electrocautery (where the tumour is burnt), biopsy forceps, or less frequently with cryoprobe (where the tumour is frozen and breaks into pieces that are removed).

\section{Outlook}

Survival rates for people with typical carcinoids are:

- $\quad 5$-year survival rate is $78-95 \%$

- 10 -year survival rate is $77-90 \%$

When a typical carcinoid is surgically removed, it is rare that the cancer will come back.

\section{Patient experiences of typical carcinoids}

"In the 2 years prior to my diagnosis, I was getting constant colds, coughs and chest infections that would take a long time to clear. I had a very bad case of pneumonia that lasted for around a week and would not go away with antibiotics. I went to my general practitioner, who referred me to a consultant.

I had some scans, which confirmed that I had a carcinoid. After that I had a bronchoscopy to have a biopsy taken, which confirmed that I had a tumour but that it was benign (would not spread). This was pretty shocking for me as the carcinoid was not affecting me on a day-to-day basis: I was going running and had a normal quality of life."

\section{Paul Harrison}

"As well as having the carcinoid removed, I had to have the right upper lobe of my lung taken out. I was in quite a lot of pain afterwards, so I would recommend that anyone else having this procedure discuss your pain medication with doctors beforehand so that you can get it right.

I am doing fine now; I am self-employed and was able to resume work about 4 months after the operation. I have organised physiotherapy for myself to help with breathing and I went back to the gym pretty soon afterwards (though I have not got back to quite the same level that I was at before). I have found that certain things, e.g. walking and talking at the same time, cause me to be more breathless than I would have been in the past, but this does not interfere with my life too much." 


\section{Atypical carcinoids}

Atypical carcinoids are less common than typical carcinoids, accounting for about $10 \%$ of all lung carcinoid tumours. They tend to grow a bit quicker than typical carcinoids and are more likely to spread to other parts of the body.

Although typical carcinoids are usually diagnosed at the earliest stage of disease (stage 1), over half are found at more advanced stages.

\section{Diagnosis}

Diagnosis of atypical carcinoids follows the same process as for typical carcinoids.

\section{Treatment}

Surgical approach is the same as for typical carcinoids; however, as atypical carcinoids are more aggressive, surgeons tend to remove bigger parts of the lung.

Chemotherapy and radiotherapy have not been shown to be very effective for treating atypical carcinoids that have spread. However, another group of medicines called somatostatin analogues, which are applied under the skin, could help with symptoms.

\section{Outlook}

The outlook for atypical carcinoids is as follows:

- 5-year survival rate: $40-60 \%$

- 10-year survival rate: $31-60 \%$

It is more common for atypical carcinoids to return after treatment than typical carcinoids; this happens in around $26 \%$ of people with an atypical carcinoid.

If you are diagnosed with a rare type of lung cancer, it is important that you ask to be referred to a specialist centre so that you can benefit from expertise in this area and also have better access to clinical trials, should you wish to be involved.

For more up-to-date information on clinical trials, you can visit:

- EU Clinical Trials Register: www.clinicaltrialsregister.eu/ctr-search/search

- ClinicalTrials.gov (from the US National Institutes of Health): https://clinicaltrials.gov

T EUROPEAN LUNG FOUNDATION

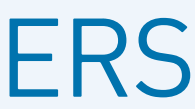

EUROPEAN RESPIRATORY SOCIETY
The European Lung Foundation (ELF) was founded by the European Respiratory Society (ERS), with the aim of bringing together patients, the public and respiratory professionals to positively influence respiratory medicine. ELF is dedicated to lung health throughout Europe, and draws together the leading European medical experts to provide patient information and raise public awareness about lung disease.

This material was compiled with the help of Dr Georgia Hardavella, Professor Bodgan Grigoriu, Dr Rohit Lal, Katharine St John-Brooks and Paul Harrison.

ECPC is the voice of the European cancer patient community, uniquely representing the interests of all cancer patient groups from the major to the rarer cancers, with more than 370 member patient organisations across Europe. More information at: www.ecpc.org

The RARECARENet (Information Network on Rare Cancers) is a European Commission-funded project that aims to build an information network to provide comprehensive information on rare cancers to the community at large. 